\title{
Effectiveness analysis of the kulback's information measure in the technological objects monitoring
}

\author{
Alexander Skatkov ${ }^{1}$, Alexey Brjuhoveckij ${ }^{1, *}$, and Dmitriy Moiseev ${ }^{2}$ \\ ${ }^{1}$ Federal Public Autonomous Educational Institution of the Higher Education «Sevastopol State University», Russian Federation, \\ 299053, Sevastopol \\ ${ }^{2}$ Federal state owned military educational institution of the Higher professional Education "the black sea higher navy order of the red star \\ academy by P.S.Nakhimov" Russian Federation, 299028, Sevastopol
}

\begin{abstract}
Annotation. The technological objects' state monitoring is focused on the following purposes: to ensure the production processes effective functioning and to improve the quality and competitiveness of the products. The aim of the proposed scientific approach is the improvement of the known statistical methods used for production processes monitoring. It allows to minimize the first and the second kind errors when evaluating the technological objects' states. The usage of Kulback's information measure allows to compress large amounts of data without losing their validity. This approach mostly satisfies the requirements for the implementation of known methods of technological objects' state monitoring. These requirements are the following: processing of large amounts of current information for setting the parameters of the monitoring and control system; real time decision-making using the monitoring results on the production process' state; timely detection of possible precritical conditions of the technological process, which can be associated with great economic damage or even catastrophic consequences.
\end{abstract}

\section{Introduction}

In modern conditions, special attention is paid to production processes' state monitoring and products' quality increasing. In addition, the scope of application of so-called critical systems and infrastructures is significantly expanding [1]. Their criticality is manifested in the fact that even small changes in parameters or structures can lead to catastrophic failures, accidents or even catastrophes. Therefore, functioning of such facilities requires the continuous operation of parametric statistical control. The main purpose of such applications lies in systematic analysis and interpretation of the processes occurring in a heterogeneous production environment. The organization of technological objects' state monitoring is associated with the following conflict situation. On the one hand - the provision of real-time mode dictates the need to use small-volume samples; on the other hand - there is a need to ensure the required reliability level of the hypotheses acceptance based on the monitoring results. Thus, it is necessary to make a compromise decision in the conditions of a priori information deficit and initial statistical uncertainty, which should guarantee risks minimization for technological objects functioning under performance requirements on speed and reliability of situation recognition procedures. Such solutions should be sought by compressing large volumes of processed data without losing their validity.

A widely used approach MSPC (Multivariate Statistical Process Control) is a natural development of the approach SPC (Statistical Process Control). According to the work [2], MSPC is a multifaceted activity associated with multifactor monitoring, modeling and process management, which is aimed at stabilizing the functioning of the enterprise, increasing its efficiency and ultimately producing high-quality products or providing quality services. One of the reasons why these control methods provide necessary but insufficient measures to obtain the final product of the proper quality is that it is necessity to control not only the quality of the final product but also the production process itself, that consists of a sequence of various operations of a complex structure.

The known solutions for operational monitoring of technological processes lies in the oil and gas industry [3]. Models and classification of monitoring systems from the viewpoint of queuing theory are considered. In the work [4] a system of monitoring of distributed technological objects is proposed. Intelligent monitoring systems for large-scale distributed technological objects are considered. A distinctive feature of such systems is the need to determine the actual global state of the system, assumed to be unchanged for the planning horizon.

\footnotetext{
* Corresponding author: bryukhovetskiy@sevsu.ru
} 
Knowledge of the actual global state of the system can be used to solve various complex optimization problems. Its representation in the system is called the information model of the state.

In [5], the author presents probabilistic statistical methods of decision theory, explaining how to detect spontaneously arising effects using observations of the realization of a random process. In conditions, including production processes, most of the problems of data analysis are related to the study of stochastic systems in which the detection of significant but rare information situations is often of decisive importance. Due to the mentioned circumstances, the known methods of production processes state monitoring cannot be a perfect operational tool, since they do not provide the required quality of control over the state of production processes. In particular, with their help it is difficult to trace the moments of changes in individual properties of the technological objects states (such as accuracy, stability, quality indicators and others). It is advisable to search for such solutions within the framework of the statistical theory of hypothesis acceptance [6]. Since, as it is well known, the possibilities of an analytical solution of such problems are very limited, it is suggested to use simulation methods as an alternative. One of the main tasks of technological objects monitoring is the synthesis of measurement metrics that can be used for the assessment of the information situation based on small samples of data. In the work [7] it is proposed to use Kulbak's distance as an information measure.

Despite a large number of practical systems used to monitor technological objects, there is an urgent need for their continuous improvement, as the complexity and scale of production systems are constantly increasing, and, consequently, the quality requirements of the products are increasing too. The main advantages of the proposed method for assessing the state of a technological facility on the basis of Kulbak's information measure are the following: sensitivity to changes in technological objects' state, low computational complexity, adaptability to external influences. Let's call the estimation of the inhomogeneity of technological object's state as J-effect, and let's call the device for its detection as J-detector. Thus, the J-effect describes instability in the production process that can lead to equipment operation violation.

\section{The proposed decision}

Software of the proposed research complex contains the following structural components:

- a subsystem for generating random samples (with a priori preset parameters close to real samples of complex systems' parameters);

- a subsystem of complex management;

- a subsystem of statistical data processing;
- a subsystem of $H_{0}$ hypothesis acceptance (about Jeffect absence) or the acceptance of competing hypothesis $H_{1}$, confirming the J-effect presence. The experiments were carried out with various combinations of input parameters, including the following:

- setting the law for the distribution of input data;

- Sample size;

- Number of intervals;

- Confidence level;

- Collection of statistical data for different time intervals;

- Estimation of the dynamics of the change in the information state of technological objects;

- Decision-making on the presence of J-effect based on a given criterion.

There is a need to compare two samples of the monitoring results to determine the significance of its qualitative change. The set of observations is a set of measurements of technological objects' states -

$X=\left\{X_{1}, X_{2}, \ldots, X_{\mathrm{N}}\right\}$, where $X_{\mathrm{i}}=\left\{x_{1}, x_{2}, \ldots, x_{\mathrm{K}}\right\}, \mathrm{N}-$ number of samples; $\mathrm{K}$ - volume of each sample; $x_{\mathrm{i}}$ measured value of technological object. The question is whether the differences between $X_{\mathrm{i}}$ and $X_{\mathrm{j}}$ can be considered significant, or the differences between them should be attributed to the random dispersion of the values of the test feature.

Kulback's information measure (Kulback's distance) between $X_{\mathrm{i}}$ and $X_{\mathrm{j}}$ can be calculated according to the rule (1):

$$
D\left(X_{i} \| X_{j}\right)=\sum_{l=1}^{K} p l \log \frac{p l}{q l},
$$

where $p_{l}$ and $q_{l}$ are respectively the probabilities of the events when values from samples $X_{\mathrm{i}}$ and $X_{\mathrm{j}}$ belong to the $l$ - interval.

Kulback's distance for distribution of $X_{\mathrm{i}}$ relatively to $X_{\mathrm{j}}$ can be calculated the following way:

- $\quad D\left(X_{i} \| X_{j}\right) \leq Q-$ J-effect absence,

- $D\left(X_{i} \| X_{j}\right)>Q-\mathrm{J}$-effect presence,

where $Q$ - distance limit (depending on the criticality of the process object).

Thus, the null hypothesis $H_{0}$ is accepted when

$$
D\left(X_{i} \| X_{j}\right) \leq Q
$$

J-effect absence - the state is stable, otherwise the hypothesis $H_{1}$ is accepted - there is a qualitative change in the state.

The problem of modeling the J-effect detection can be solved using the following algorithmic scheme:

1) $N$ samples of $X_{\text {i }}$ of a given volume $K$ are generated from a population having a predetermined distribution law;

2) Two samples are selected $\left(X_{\mathrm{i}}\right.$ and $\left.X_{\mathrm{j}}\right)$ to determine the Kulback's distance; 
3) Confidence level is given depending on the criticality of the technological object;

4) The $H_{0}$ hypothesis is formulated;

5) The calculated criterion value $Q$ is determined (at a given confidence level to test the null hypothesis);

6) Critical areas of values of the criterion for testing the null hypothesis are determined - areas of stable / unstable J-effect detection;
7) For the specified number of times the algorithmic points $(2-6)$ are repeated for other pairs of samples $X_{\mathrm{i}}$ and $X_{\mathrm{j}}$. Then a conclusion is made about an assessment of their differences.

On the fig. 1 the main moduls of the software system are presented.

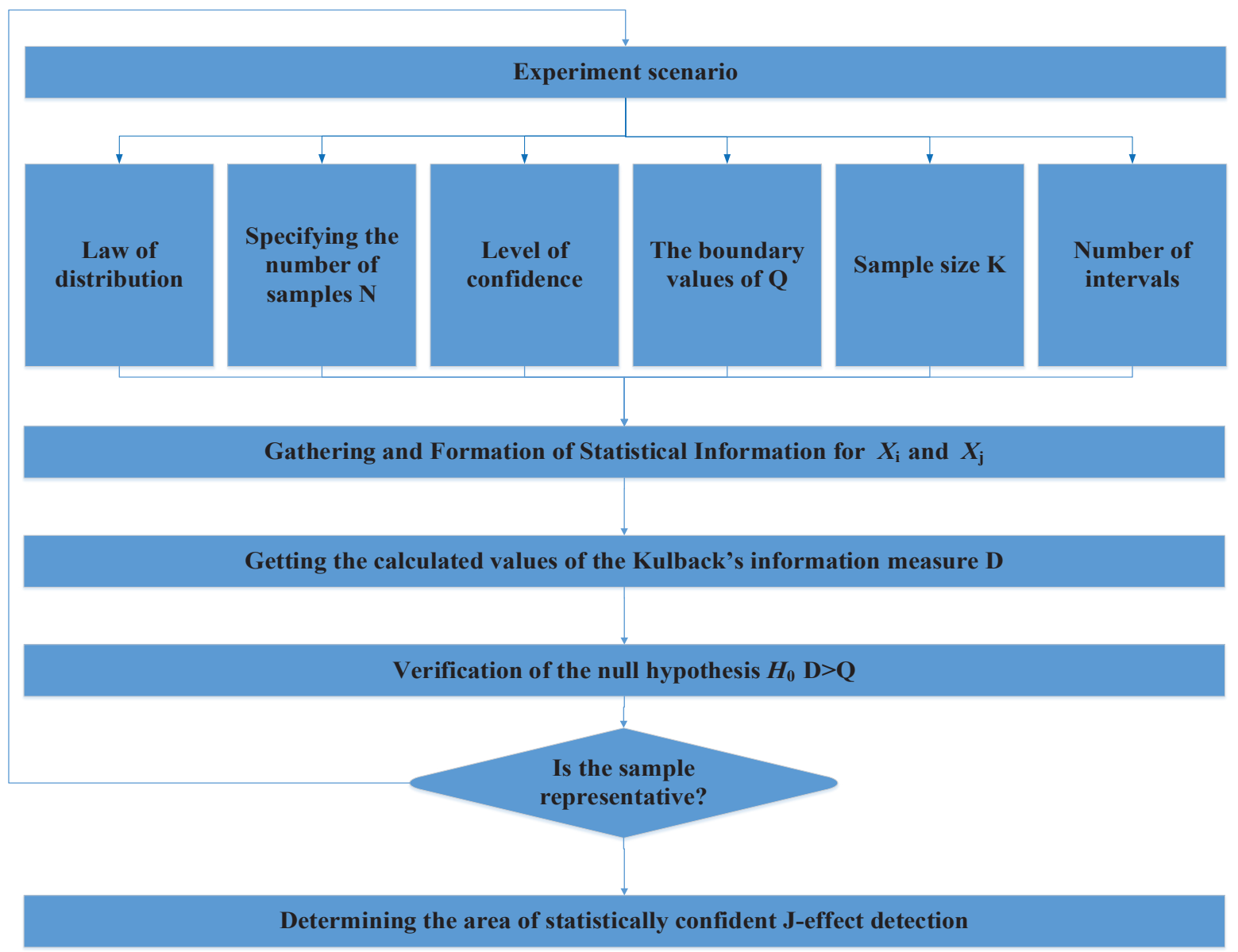

Fig. 1. Block diagram of the J-effect detection software system

The graphical programming environment Simulink from the package MATLAB have been used to illustrate the process of technological objects' states changing detection.

The process of J-effect detection based on using of Kulback's information measure can be considered on the following example. The task is to control the $\lambda$ parameter that is distributed according to the normal law with mathematical expectation $M(\lambda)$ and the standard deviation $\sigma(\lambda)$.

The experiments have been carried out to answer the following question: what effect can the parameters $K, N$ and $\sigma(\lambda)$ render on the changes in the states of technological objects and on the definition of stable recognition areas of J-effect. The following settings has been used:
- Sample sizes varied in the ranges: $50<=\mathrm{K}<=5000$; - Number of samples $X=\left\{X_{1}, X_{2}, \ldots, X_{\mathrm{N}}\right\}, N=4,7,10$; - Expected value $M(\lambda)=1$;

$-\sigma(\lambda)$ varied in the range $[0,05 ; 0,35]$ in increments of $\Delta \sigma(\lambda)=0,05$.

In order to ensure the universality of the measured values processing and independence from the controlled metrics the software system normalizes the values of the samples $X_{\mathrm{j}}$ according to the rule (2).

$$
x_{\mathrm{inorm}}=\left(x_{\mathrm{i}}-\mathrm{M}_{\mathrm{x}}\right) / \mathrm{M}_{\mathrm{x}},
$$

\section{Results of experiments}

The results of the experiment are presented on the fig. 2 . The following settings were used: 
Sample size $K=5000,500,50$;

Number of samples $N=7$;

$M(\lambda)=1$;

$\sigma(\lambda)=[0,05 ; 0,1 ; 0,15 ; 0,2 ; 0,25 ; 0,3 ; 0,35]$.

A series of experiments was performed to assess Jeffect. The values of the following samples were compared:

$$
X_{1}-X_{2}, X_{1}-X_{3}, \ldots, X_{1}-X_{7} \text {. }
$$

Thus, the following patterns in J-effect detection were established as a result of simulated experiments.

1) Stable J-effect detection for the technological objects with the given input parameters is observed at the sample size $K>500$ and $\sigma(\lambda) \geq 0,15$.

2) When using the sample size $K \leq 80 \mathrm{~J}$-effect is unstable in the range от $0,15 \leq \sigma(\lambda) \leq 0,3$.

3) J-effect stably is not observed when $K<50$ over the entire range of variation $\sigma(\lambda)$.

4) During the experiments it was found that the higher the value $\Delta \sigma(\lambda)$ is, the more effectively the quality status is monitored technological objects. Thus, the maximum Jeffect is observed when comparing the results of experiments obtained for samples $X_{1}-X_{7}$.

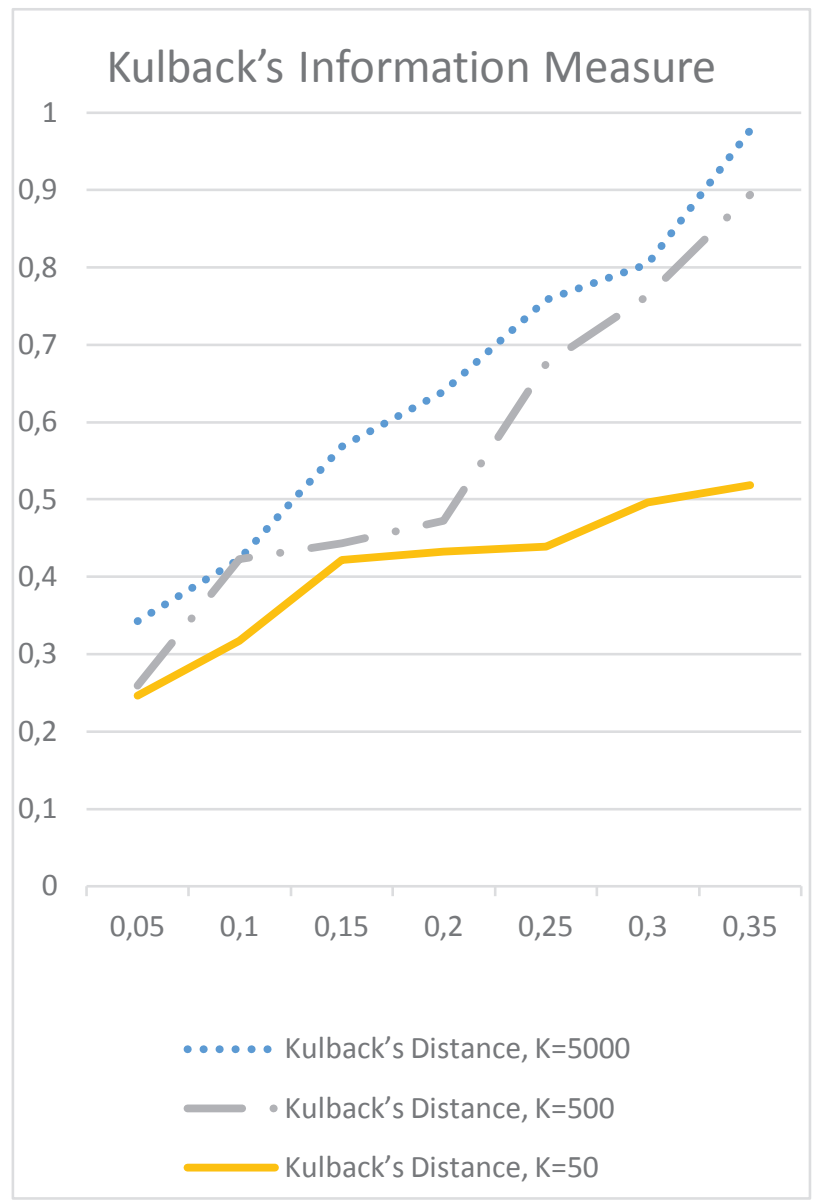

Fig. 2. The results of the experiments on the J-effect presence assessment

The obtained results shows that the application of the model on the basis of Kulbak's information measure allows to provide statistical stability of the area of confident / uncertain J-effect detection when monitoring the qualitative changes in the state of technological objects. The general recommendation for assessing detection is as follows. Depending on the purpose of the monitored system (is it critical or not), the decision-maker is authorized to set the optimal values of confidence level $-p$ and the corresponding value of distance limit $Q$. There are two goals: Ensure a high reliability of the values of the characteristics of the monitored process facilities, to minimize the production losses. The results obtained in the conducted experiments with models are used as a basis for information technology for monitoring the technological objects' state and are used as input to the intellectual decision support system. This system is to ensure the minimal losses that can be caused by:

- untimely decision-making (cost of information loss),

- $\quad$ state control costs.

Each type of loss is compared with the coefficient $-\alpha$ and $\beta$ respectfully: $\alpha+\beta=1$. This system contains evaluation matrix, the elements of which are the values of losses, and inputs - threshold values of Kulbak's distance$Q_{m}, m$ - Number of gradations of $Q ; f_{t}$ - frequency of measurement of process object control parameters and values $\sigma(\lambda)$.

In the plans for further research, it is proposed to evaluate the complex influence of the input parameter values on statistical sensitivity, statistical stability, areas of confident / uncertain detection under other distribution laws of the parameters under study, for a different number of intervals, and to build a decision support system for detecting the J-effect.

This work was partially supported by the Russian Foundation for Basic Research (project № 15-29-07936).

\section{References}

1. A. Skatkov, Information technology for critical infrastructures, 306, (2012)

2. I. Kablashova, Theory and methods of ensuring the quality of production processes, 173, (2005)

3. E Ohotnikov, Bulletin of Tyumen State University, 7, 80, (2006)

4. S. Andryushkevich, Bulletin of the Tomsk Polytechnic University, 317, 35, (2010)

5. A. Shiryaev, Probabilistic-statistical methods in the theory of decision-making, 144, (2014)

6. S. Ayvazyan, Statistics and the Foundations of Econometrics, 1000, (1998)

7. S. Kulback, Information theory and statistics, 408, (1967) 